\title{
Nonlinear Singular First Order Partial Differential Equations Whose Characteristic Exponent Takes a Positive Integral Value
}

\author{
By
}

\author{
Hideshi YAmANE*
}

\begin{abstract}
We consider nonlınear singular partıal differential equations of the form $\left(t D_{t}-\rho(x)\right) u=t a(x)$ $+G_{2}(x)\left(t, t D_{t} u, u, D_{1} u, \ldots, D_{n} u\right)$.

It has been proved by Gerard and Tahara that there exists a unique holomorphic solution with $u(0, x) \equiv 0$ if the characteristic exponent $\rho(x)$ avoids positive integral values. In the present paper we consider what happens if $\rho(x)$ takes a positive integral value at $x=0$. Generically, the solution $u(t, x)$ is singular along the analytic set $\left\{t=0, \rho(x) \in \mathbb{N}^{*}\right\}, \mathbb{N}^{*}=\{1,2, .$.$\} , and we investigate how$ far it can be analytically continued.
\end{abstract}

\section{§1. Introduction}

In this paper we consider the following type of nonlinear singular partial differential equations:

$$
\left(t D_{t}-\rho(x)\right) u=t a(x)+G_{2}(x)\left(t, t D_{t} u, u, D_{1} u, \ldots, D_{n} u\right),
$$

where $(t, x) \in \mathbb{C}_{t} \times \mathbb{C}_{x}^{n}, x=\left(x_{1}, \ldots, x_{n}\right), D_{t}=\partial / \partial t, D_{\imath}=\partial / \partial x_{\imath}$. We assume that $\rho(x)$ and $a(x)$ are holomorphic functions defined in a polydisk $D$ centered at the origin of $\mathbb{C}_{x}^{n}$ and

$$
G_{2}(x)\left(t, z, X_{0}, X_{1}, \ldots, X_{n}\right)=\sum_{p+q+|\alpha| \geq 2} a_{p q \alpha}(x) t^{p} z^{q} X_{0}^{\alpha_{0}} \cdots X_{n}^{\alpha_{n}},|\alpha|=\alpha_{0}+\cdots+\alpha_{n} .
$$

Here $a_{p q \alpha}(x)$ is holomorphic in $D$ and $\sum_{p+q+|\alpha| \geq 2} \sup _{x \in D}\left|a_{p q \alpha}(x)\right| t^{p} z^{q} X_{0}^{\alpha_{0}} \cdots X_{n}^{\alpha_{n}}$ is a convergent power series in $\left(t, z, X_{0}, \ldots, X_{n}\right)$.

Now we look for a (necessarily unique) local holomorphic solution $u(t, x)$ with $u(0, x) \equiv 0$. The right hand side of $(1)$ is well-defined because of this

Communicated by T. Kawa1, April 7, 1997.

1991 Mathematics Subject Classifications 35A20

* Department of Mathematics, Chiba Institute of Technology. Shibazono, Narashino 275-0023, Japan

E-mail: yamane@cc.it-chıba.ac.jp 
initial condition.

The following theorem is proved in [1].

Theorem 1 (Gérard-Tahara). Let $\stackrel{0}{0} \neq 0$ be a point in D. If $\rho\left(\stackrel{\circ}{x} \notin \mathbb{N}^{*}=\right.$ $\{1,2,3, \ldots\}$, then the equation (1) has a unique holomorphic solution $u(t, x)$ with $u(0, x) \equiv 0$ in a neighborhood of $(0, \dot{x}) \in \mathbb{C}_{t} \times \mathbb{C}_{x}^{n}$.

In this paper we consider what happens if $\rho(\stackrel{\circ}{x})$ takes a positive integral value. series:

First we explain the calculation in [1]. They express $u(t, x)$ as a power

$$
u(t, x)=\sum_{m \geq 1} u_{m}(x) t^{m}
$$

Then $\left\{u_{m}(x)\right\}$ satisfies the following recurrence formula:

$$
u_{1}(x)=\frac{a(x)}{1-\rho(x)}
$$

and for $m \geq 2$

$$
\begin{aligned}
& (m-\rho(x)) u_{m}(x) \\
& =f_{m}\left(u_{1}(x), 2 u_{2}(x), \ldots,(m-1) u_{m-1}(x), u_{1}(x) \ldots, u_{m-1}(x),\right. \\
& \left.\quad D_{1} u_{1}, \ldots, D_{n} u_{1} \ldots ., D_{1} u_{m-1}, \ldots, D_{n} u_{m-1},\left\{a_{p q \alpha}(x)\right\}_{p+q+|\alpha| \leq m}\right) .
\end{aligned}
$$

Here $f_{m}$ is a polynomial whose coefficients are 1 .

The assumption $\rho\left(x^{\circ}\right) \notin \mathbb{N}^{*}$ guarantees that $u_{m}(x)$ is holomorphic in a common neighborhood of the origin of $\mathbb{C}_{x}^{n}$ for all $m$. On the other hand, if $\rho(\dot{x})$ $\in \mathbb{N}^{*}$ then $u_{m}(x)$ may be singular at $x=x^{\circ}$ for some $m$, and there exists no holomorphic solution with $u(0, x) \equiv 0$ in any neighborhood of $(0, \dot{x}) \in \mathbb{C}_{t} \times \mathbb{C}_{x}^{n}$. This situation is what we would like to consider in this paper.

Example. The equation

$$
\left(t D_{t}-\left(1-x^{g}\right)\right) u=t x^{h}+G_{2}(x)\left(t, t D_{t} u, u, D_{1} u, \ldots, D_{n} u\right), \quad g, h \in \mathbb{N}^{*}
$$

has a (unique) holomorphic solution $u(t, x)=\sum_{m \geq 1} u_{m}(x) t^{m}$ if and only if $g \leq h$.

Remark. It sometimes happens, as is shown in the example above, that $\imath_{m}(x)$ determined by (3) and (4) is holomorphic for all $m$ even when $\rho(0) \in$ $\mathbb{N}^{*}$. In [1] it is proved that $u(t, x)=\sum_{m \geq 1} u_{m}(x)$ is convergent in a neighborhood of the origin in such a case.

Now we assume the following:

$$
\rho(0) \in \mathbb{N}^{*}=\{1,2,3, \ldots\}, \quad \rho(x) \not \equiv \rho(0) .
$$

Under this assumption, the set $V=\{\rho(x)=\rho(0)\} \subset \mathbb{C}_{x}^{n}$ is an analytic set of codimension 1. The equation (1) has a unique holomorphic solution $u(t, x)$ 
with $u(0, x) \equiv 0$ outside $V$, and there may be no such solution near any point in $V$. Now we put

$$
d(x)=\operatorname{dist}(x, V \cup \partial D)=\operatorname{dist}(x, V),
$$

where dist $(x, Z)$ is the distance from $x$ to a subset $Z \subset \mathbb{C}_{x}^{n}$. The second equality holds if $x$ belongs to a sufficiently small neighborhood of the origin. We claim that the solution $\imath(t, x)$ is holomorphic in an open set of the following form:

$$
|t|<C d(x)^{p}, \quad x \text { is sufficiently close to the origin, }
$$

where $p$ and $C$ are positive constants. A strinking feature of $p$ is that it is completely determined by $\rho(x)$ and nothing else. A more precise statement will be given later.

Assume that $\rho(x)-\rho(0)$ has a zero exactly of order $g$ at $x=0$. Then we have the following estimate:

$$
\left|\frac{1}{\rho(x)-\rho(0)}\right| \leq C^{\prime} d(x)^{-g} .
$$

where $C^{\prime}$ and $g$ are positive constants. The proof of this estimate will be given in Appendix.

Now we announce

Theorem 2 (Main Theorem).

(1) If $\rho(0) \geq g+2$, then the solution $u(t, x)$ of (1) with $u(0, x) \equiv 0$ is holomorphic in a domain of the form

$$
|t|<C d(x) \text {. }
$$

(ii) If $\rho(0)<g+2$, then the solution $u(t, x)$ of (1) with $u(0, x) \equiv 0$ is holomorphic in a domain of the form

$$
|t|<C d(x)^{\frac{g+2}{\rho(0)}}
$$

In both cases $C$ is a constant $>0$ determined by $\rho(x), a(x)$ and $G_{2}\left(t, z, X_{0}\right.$, $\left.X_{1}, \ldots, X_{n}\right)$.

\section{\$2. Proof of the Main Theorem}

We express the solution $u(t, x)$ in the form of a power series in $t$ :

$$
u(t, x)=\sum_{m=1}^{\infty} u_{m}(x) t^{m}
$$

Then $\left\{u_{m}(x)\right\}$ satisfies the following recursive formula:

$$
u_{1}(x)=\frac{a(x)}{1-\rho(x)}
$$


and for $m \geq 2$

$$
\begin{aligned}
& (m-\rho(x)) u_{m}(x) \\
& =f_{m}\left(u_{1}(x), 2 u_{2}(x), \ldots,(m-1) u_{m-1}(x), u_{1}(x), \ldots, u_{m-1}(x),\right. \\
& \left.\quad D_{1} u_{1}, \ldots, D_{n} u_{1}, \ldots, D_{1} u_{m-1}, \ldots, D_{n} u_{m-1},\left\{a_{p q \alpha}(x)\right\}_{p+q+|\alpha| \leq m}\right) .
\end{aligned}
$$

Here $f_{m}$ is a polynomial whose coefficients are 1 . Put $\rho(0)=M \in \mathbb{N}^{*}=\{1,2, \ldots\}$. Then $u_{m}(x)(m \geq M)$ may be singular along $V=\left\{x \in \mathbb{C}^{n} ; \rho(x)=M\right\}$. It is easy to see that we have the following type of estimate:

$$
\left|u_{m}(x)\right| \leq C_{m} d(x)^{-s_{m}} \quad(m \geq M)
$$

in a common neighborhood of the origin, where $C_{m}$ is a positive constant and $s_{m}$ $(m \geq M)$ is a positive integer. Obviously we can take $s_{M}=g$. (The first $M-1$ terms $s_{1}, \ldots, s_{M-1}$ will be given later in a technical fashion).

We have

Proposition $\mathbb{1}$. We can choose $s_{m}=m+g-M(m \geq M)$ if $M \geq g+2$. On the other hand, we can choose $s_{I M+k}=l(g+2)+k-2(l \geq 1,0 \leq k \leq M-1)$ if $M<g+2$.

Proof.

Obviously we have

$$
\left|D_{k} u_{m}(x)\right| \leq C_{m}^{\prime} d(x)^{-\left(s_{m}+1\right)}, \quad m \geq M, k=1, \ldots, n
$$

for some positive constant $C_{m}^{\prime}$. Hence we may set, for $m \geq M+1$,

$$
\begin{aligned}
s_{m}=\max & {\left[\left\{s_{m_{1}}+1 ; 1 \leq m_{1} \leq m-1\right\}\right.} \\
& \left.\cup\left\{\sum_{j^{\prime}=1}^{j}\left(s_{m,}+1\right) ; 2 \leq j \leq m, m_{j^{\prime}} \geq 1\left(1 \leq j^{\prime} \leq j\right), \sum_{j^{\prime}=1}^{j} m_{j^{\prime}} \leq m\right\}\right] .
\end{aligned}
$$

Here we set $s_{m}=-1(1 \leq m \leq M-1)$. This technical choice is made in order to deal with the exceptional cases $m=1, \ldots, M-1$ where $u_{m}$ is holomorphic and so $\imath_{m}$ and its derivatives are bounded. In these cases we don't need the kind of estimate like (10), in which the term +1 was necessary because of the singularity of $u_{m}(m \geq M)$. The quantity $s_{m_{1}}+1$ comes from $G_{2}$ 's terms which are linear in $u_{1}, \ldots, u_{m-1}, D_{1} u_{1}, \ldots, D_{n} u_{1}, \ldots, D_{1} u_{m-1}, \ldots, D_{n} u_{m-1}$ and $\sum_{j^{\prime}=1}^{j}\left(s_{m g^{\prime}}+1\right)$ comes from $G_{2}$ 's $j$-th degree terms in $u_{1}, \ldots, u_{m-1}, D_{1} u_{1}, \ldots, D_{n} u_{1} \ldots, D_{1} u_{m-1}, \ldots$, $D_{n} u_{m-1}$.

We can simplify (11) slightly. Since $s_{m} \geq s_{m-1}+1(m \geq M+1)$ follows immediately from (11), we have $s_{m} \geq s_{m-1}(m \geq 2)$. Hence we obtain

$$
s_{m}=\max \left[\left\{s_{m-1}+1\right\} \cup\left\{\sum_{j^{\prime}=1}^{\prime}\left(s_{m,,^{\prime}}+1\right) ; 2 \leq j \leq m, m_{j^{\prime}} \geq 1\left(1 \leq j^{\prime} \leq j\right), \sum_{j^{\prime}=1}^{\prime} m_{j^{\prime}}=m\right\}\right] \text {. }
$$

Moreover, by using the fact 


$$
\left\{s_{m-1}+1\right\} \subset\left\{\left(s_{m_{1}}+1\right)+\left(s_{m_{2}}+1\right) ; m_{1} \geq 1, m_{2} \geq 1, m_{1}+m_{2}=m\right\}
$$

we obtain

$$
s_{m}=\max _{j=2, \ldots, m}\left\{\sum_{j^{\prime}=1}^{j}\left(s_{m, \prime}+1\right) ; m_{1} \geq 1, \ldots, m_{j} \geq 1, \sum_{j^{\prime}=1}^{j} m_{j^{\prime}}=m\right\} .
$$

Now we prove the case $M \geq g+2$ by induction on $m$. The desired formula is obviously true for $m=M$. Assume that the formula is true for $s_{1}, \ldots, s_{m-1}$, where $m \geq M+1$. Then we have

$$
s_{m-1}+1=\{(m-1)+g-M\}+1=m+g-M .
$$

The proof will end when we prove that $s_{m-1}+1$ attains the maximum in the right hand side of (12) by using the following iequality:

$$
\begin{aligned}
& \sum_{j^{\prime}=1}^{j}\left(s_{m_{j^{\prime}}}+1\right) \leq \sum_{j^{\prime} \in A} m_{j^{\prime}}+(\operatorname{card} A)(g-M+1), \\
& A \stackrel{\text { def }}{=}\left\{j^{\prime} ; m_{j^{\prime}} \geq M\right\} \subset\{1, \ldots, j\} .
\end{aligned}
$$

If $A=\phi$, then $\sum_{j^{\prime}=1}^{j}\left(s_{m, r}+1\right)=0 \leq m+g-M$, where the last inequality follows from the assumption $m \geq M+1$.

Next if $\operatorname{card} A=1$, we have, since each $m_{\jmath^{\prime}} \geq 1$,

$$
\sum_{j^{\prime} \in A} m_{j^{\prime}} \leq m-\operatorname{card} A^{c}=m-j+1 .
$$

Here we have used the notation $A^{c}=\{1,2, \ldots, j\} \backslash A$. So it follows from (13)

$$
\begin{aligned}
\sum_{j^{\prime}=1}^{j}\left(s_{m,,^{\prime}}+1\right) & \leq m-j+1+(g-M+1) \\
& =m+g-M-j+2 \\
& \leq m+g-M,
\end{aligned}
$$

because $j \geq 2$.

Last, if $\operatorname{card} A \geq 2$,

$$
\begin{aligned}
\sum_{j^{\prime} \in A} m_{j^{\prime}}+(\operatorname{card} A)(g-M+1) & \leq m+2(g-M+1) \\
& \leq m+g-M,
\end{aligned}
$$

because $g-M \leq-2$.

The case $M \geq g+2$ has now been proved.

Next, we prove the case $M<g+2$. First we assume that $l=1$. The case $k=0$ obviously holds. It is easy to see that $s_{m}=s_{m-1}+1, M+1 \leq m \leq 2 M-1$. Hence the case $l=1$ is proved.

Now suppose that the claim has been shown for $s_{1}, s_{2}, \ldots, s_{l M+k-1}, l \geq 2,0 \leq k$ $\leq M-1$. Then we have

$$
\begin{aligned}
\left(s_{(l-1) M}+1\right)+\left(s_{M+k}+1\right) & =\{(l-1)(g+2)-1\}+\{(g+2)+k-1\} \\
& =l(g+2)+k-2 .
\end{aligned}
$$


We are going to prove that this attains the maximum of the right hand side of (12). When $l_{1}+\cdots+l_{j}=l-l^{\prime}, k_{1}+\cdots+k_{j}=M l^{\prime}+k$,

$$
\begin{aligned}
\left(s_{l_{1} M+k_{1}}+1\right)+\cdots+\left(s_{l, M+k}+1\right) & =\sum_{j^{\prime} \in A}\left\{l_{j^{\prime}}(g+2)+k_{j^{\prime}}-1\right\} \\
& =(g+2) \sum_{j^{\prime} \in A} l_{j^{\prime}}+\sum_{j^{\prime} \in A} k_{j^{\prime}}-\operatorname{card} A
\end{aligned}
$$

for $A=\left\{j^{\prime} ; l_{j^{\prime}} \geq 1\right\} \subset\{1,2, \ldots, j\}$. Remark that $\sum_{j^{\prime} \in A} l_{j^{\prime}}=\sum_{j^{\prime}=1}^{j_{j^{\prime}}}=l-l^{\prime}$ and that $k_{j^{\prime}} \geq 1$ if $l_{j^{\prime}}=0$. If $A=\phi$, then the right hand side is equal to $0 \leq l(g+2)+k-2$. The claim obviously holds in this case.

Next if $A \neq \phi$, then

$$
\begin{aligned}
& \left(s_{l_{1} M+k_{1}}+1\right)+\cdots+\left(s_{l, M+k}+1\right) \\
= & \left(l-l^{\prime}\right)(g+2)+\sum_{j^{\prime} \in A} k_{g^{\prime}}-\operatorname{card} A .
\end{aligned}
$$

Let us estimate the second term in the right hand side. If $j^{\prime} \notin A$ then $l_{j^{\prime}}=0$ and $k_{J^{\prime}} \geq 1$. So it follows that

$$
\begin{aligned}
\sum_{j^{\prime} \in A} k_{j^{\prime}} & =\left(M l^{\prime}+k\right)-\sum_{j^{\prime} \notin A} k_{j^{\prime}} \\
& \leq\left(M l^{\prime}+k\right)-\operatorname{card} A^{c} \\
& =\left(M l^{\prime}+k\right)-(j-\operatorname{card} A) .
\end{aligned}
$$

By combining (14) and (15) we obtain

$$
\begin{aligned}
& \left(s_{l_{1 M+k 1}}+1\right)+\cdots+\left(s_{l, M+k}+1\right) \\
\leq & \left(l-l^{\prime}\right)(g+2)+\left(M l^{\prime}+k\right)-j \\
= & l(g+2)+l^{\prime}\{-(g+2)+M\}+k-j \\
\leq & l(g+2)+k-j \\
\leq & l(g+2)+k-2 .
\end{aligned}
$$

Thus we have proved that $s_{l M+k}=l(g+2)+k-2$.

We will make use of the following

Lemma 1. Let $\Omega$ be a domain in $\mathbb{C}_{x}^{n}, x=\left(x_{1}, \ldots, x_{n}\right)$, and assume that a holomorphic function $u(x)$ in $\Omega$ satisfies

$$
|u(x)| \leq \frac{C(r)}{r^{a}}, \quad a \in \mathbb{N}=\{0,1,2, \ldots\}
$$

where $r=\operatorname{dist}(x ; \partial \Omega)$ is the distance from $x$ to the boundary $\partial \Omega$ of $\Omega$ and $C(r)$ is a polynomial in $r$ of degree $\leq a$ with non-negative coefficients. Then we have

$$
\left|D_{i} u(x)\right| \leq \frac{e(a+1) C(r)}{r^{a+1}}, \quad i=1, \ldots, n .
$$


Proof.

We may assume that $i=1$ without loss of generality. Goursat's formula implies that

$$
D_{1} u(x)=\frac{1}{2 \pi \sqrt{-1}} \oint_{\Gamma} \frac{u\left(y, x_{2}, \ldots, x_{n}\right)}{\left(x_{1}-y\right)^{2}} d y .
$$

where $\Gamma=\left\{\left|y-x_{1}\right|=\frac{1}{a+1} r\right\} \subset \mathbb{C}_{y}$. Since

$$
\operatorname{dist}\left(\left(y, x_{2}, \ldots, x_{n}\right) ; \partial \Omega\right) \geq r-\frac{r}{a+1}=\frac{a}{a+1} r \quad \text { for } \quad y \in \Gamma \text {, }
$$

we have by writing $C(r)=\sum_{\jmath=0}^{a} C_{\jmath} r^{\jmath}$.

$$
\begin{aligned}
\sup _{y \in \Gamma}\left|u\left(y, x_{2}, \ldots, x_{n}\right)\right| & \leq \sum_{j=0}^{a} C_{j}\left(\frac{a}{a+1} r\right)^{\jmath-a} \\
& \leq\left(\frac{a}{a+1}\right)^{-a} \sum_{j=0}^{a} C_{\jmath} r^{j-a} \leq e \frac{C(r)}{r^{a}}
\end{aligned}
$$

Hence we get the following estimate:

$$
\begin{aligned}
\left|D_{1} u(x)\right| & \leq \frac{1}{2 \pi} \cdot 2 \pi \frac{r}{a+1} \cdot \frac{1}{\left(\frac{1}{a+1} r\right)^{2}} \cdot e \frac{C(r)}{r^{a}} \\
& =\frac{e(a+1) C(r)}{r^{a+1}} .
\end{aligned}
$$

Now we come back to the proof of the Main Theorem.

In a sufficiently small neighborhood of the origin, we may assume that the following estimates hold:

$$
\begin{aligned}
& \left|j u_{j}(x)\right| \leq A, \quad\left|D_{i},(x)\right| \leq A, \quad(j=1, \ldots, M-1 . i=1, \ldots, n), \\
& \left|u_{M}(x)\right| \leq A d(x)^{-g}, \quad\left|D_{i} u_{M}(x)\right| \leq A d(x)^{-(g+1)}, \quad(i=1, \ldots, n), \\
& \frac{s_{m}+1}{N m} \leq 1(m \geq M) . \quad|m-\rho(x)| \geq N \sigma m(m \geq M+1), \\
& \left|a_{p q \alpha}(x)\right| \leq A_{p q \alpha},
\end{aligned}
$$

where $A . N, \sigma$ and $A_{p q \alpha}$ are positive constants and $\sum_{p+q+|\alpha| \geq 2} A_{p q \alpha} t^{p} z^{q} X_{0}^{\alpha_{0}} X_{1}^{\alpha_{1}} \cdots X_{n}^{\alpha_{n}}$ is a convergent power series.

Consider now the following analytic equation (with parameter $d>0$ ):

$$
\begin{aligned}
\sigma Y= & \sigma\left(A t+A t^{2}+\cdots+A t^{M-1}+\frac{A}{d^{g+1}} t^{M}\right) \\
& +\frac{1}{d} \sum_{p+q+|\alpha| \geq 2} A_{p q \alpha} t^{p} Y^{q} Y^{\alpha_{0}}(e Y)^{\alpha_{1}} \cdots(e Y)^{\alpha_{n}} \\
& -\frac{1}{d} \sum_{m=2}^{M} B_{m} t^{m},
\end{aligned}
$$


where $B_{m}$ is the coefficient defined by the following identity:

$$
\begin{gathered}
\sum_{m=2}^{\infty} B_{m} t^{m}=\sum_{p+q+|\alpha| \geq 2} A_{p q \alpha} e^{\alpha_{1}+\cdots+\alpha_{n}} t^{p}\left(A t+A t^{2}+\cdots+A t^{M-1}\right)^{q+|\alpha|}, \\
|\alpha|=\alpha_{0}+\left(\alpha_{1}+\cdots+\alpha_{n}\right) .
\end{gathered}
$$

By the implicit function theorem, this analytic equation has a unique holomorphic solution $Y$ of the form

$$
Y=\sum_{m \geq 1} Y_{m}(d) t^{m}
$$

Here $Y_{m}(d)$ is determined by the following type of recursive formula:

$$
Y_{1}=\cdots=Y_{M-1}=A, \quad Y_{M}=\frac{A}{d^{g+1}}
$$

and for $m \geq M+1$,

$$
\sigma Y_{m}=\frac{1}{d} F_{m}\left(Y_{1}, \ldots, Y_{m-1} ; e Y_{1}, \ldots, e Y_{m-1} ;\left\{A_{p q \alpha}\right\}_{p+q+|\alpha| \leq m}\right),
$$

where $F_{m}$ is a polynomial with positive coefficients.

It is easy to see that $Y_{m}(d)$ is of the form

$$
Y_{m}(d)=\frac{C_{m}(d)}{d^{t_{m}}},
$$

where $C_{m}$ is a polynomial of order $\leq t_{m}$ with non-negative coefficients. Here $t_{m}=0(1 \leq m \leq M-1), t_{M}=g+1$ and for $m \geq M+1$

$$
\begin{aligned}
t_{m}=1+\max & {\left[\left\{t_{m_{1}} ; 1 \leq m_{1} \leq m-1\right\}\right.} \\
& \left.\cup\left\{\sum_{j^{\prime}=1}^{j} t_{m, i}: 2 \leq j \leq m, m_{j^{\prime}} \geq 1\left(1 \leq j^{\prime} \leq j\right), \sum_{j^{\prime}=1}^{j} m_{j^{\prime}} \leq m\right\}\right] .
\end{aligned}
$$

It is obvious that $t_{m}=s_{m}+1(m \geq 1)$. So we have

$$
\begin{aligned}
t_{m} & =m+g-M+1(m \geq M) \text { if } M \geq g+2 . \\
t_{l M+k} & =l(g+2)+k-1(l \geq 1,0 \leq k \leq M-1) \text { if } M<g+2 .
\end{aligned}
$$

We are going to prove that $Y$ is a majorant power series of $u$ if $d=d(x)$. More precisely. we want to show that for $m \geq 1$

$$
\begin{gathered}
\left|u_{m}(x)\right| \leq\left|m u_{m}(x)\right| \leq Y_{m}(d) \\
\left|D_{i} u_{m}(x)\right| \leq_{e} Y_{m}(d), \quad i=1,2, \ldots, n .
\end{gathered}
$$

The cases $m=1,2, \ldots, M$ are obviously true. We will prove the remaining cases by induction on $m$. Suppose that the above inequalities have been shown to be true for $u_{1}, u_{2}, \ldots, u_{m-1}$. Then we have 


$$
\begin{aligned}
& \left|u_{m}(x)\right| \\
& \leq \frac{1}{|m-\rho(x)|} f_{m}\left(\left|u_{1}\right|, 2\left|u_{2}\right|, \ldots,(m-1)\left|u_{m-1}\right|,\left|u_{1}\right|,\left|u_{2}\right|, \ldots\left|u_{m-1}\right|,\right. \\
& \left.\left|D_{1} u_{1}\right|, \ldots,\left|D_{n} u_{1}\right|, \ldots,\left|D_{1} u_{m-1}\right|, \ldots,\left|D_{n} u_{m-1}\right| ;\left\{\left|a_{p q \alpha}\right|\right\}_{p+q+\alpha \leq m}\right) \\
& \leq \frac{1}{N \sigma m} f_{m}\left(\left|u_{1}\right|, 2\left|u_{2}\right|, \ldots,(m-1)\left|u_{m-1}\right|,\left|u_{1}\right|,\left|u_{2}\right|, \ldots\left|u_{m-1}\right|\right. \text {, } \\
& \left.\left|D_{1} u_{1}\right|, \ldots,\left|D_{i \imath} u_{1}\right|, \ldots,\left|D_{1} u_{m-1}\right|, \ldots,\left|D_{n} u_{m-1}\right| ;\left\{\left|a_{p q \alpha}\right|\right\}_{p+q+\alpha \leq m}\right) \\
& \leq \frac{1}{N \sigma m} f_{m}\left(Y_{1}, Y_{2}, \ldots, Y_{m-1}, Y_{1}, Y_{2}, \ldots, Y_{m-1}\right. \text {, } \\
& \left.e Y_{1}, \ldots, e Y_{1}, \ldots, c Y_{m-1}, \ldots, e Y_{m-1} ;\left\{A_{p q \alpha}\right\}_{p+q+\alpha \leq m}\right) \\
& =\frac{1}{N \sigma m} F_{m}\left(Y_{1}, \ldots, Y_{m-1}, e Y_{1}, \ldots, e Y_{m-1} ;\left\{A_{p q \alpha}\right\}_{p+q+\alpha \leq m}\right) \quad \text { (It's an equality!) } \\
& =\frac{1}{N \sigma m} \cdot \sigma d Y_{m}(d)=\frac{d}{N m} Y_{m}(d) \text {. }
\end{aligned}
$$

Therefore we obtain

$$
\left|m \imath_{m}(x)\right| \leq \frac{d}{N} Y_{m}(d) \leq Y_{m}(d) .
$$

Here we assume that $x$ is in a ball of radius $<N$ centered at the origin. Hence $0<d<N$. Moreover, since

$$
\left|u_{m}(x)\right| \leq \frac{1}{N m} d Y_{m}(d)=\frac{1}{N m} \frac{C_{m}(d)}{d^{t_{m}-1}} .
$$

we deduce by using the lemma that

$$
\left|D_{\imath} u_{m}(x)\right| \leq \frac{t_{m}}{N m} e \frac{C_{m}(d)}{d^{t_{m}}} \leq e \frac{C_{m}(d)}{d^{t_{m}}}=e Y_{m}(d) .
$$

So induction proceeds. We have proved that $u \ll Y$.

Our next investigation is about the convergence of $Y=\sum_{m \geq 1} Y_{m}(d) t^{m}$ and $u(t . x)=\sum_{m \geq 1} u_{m}(x) t^{m}$.

Fix some sufficiently small $d_{0}>0$. Then for some $T>0$, the series $\sum_{m \geq 1} Y_{m}\left(d_{0}\right) T^{m}$ is convergent by the implicit function theorem. have

Let us consider the case $M \geq g+2$, where $t_{m}=m+g-M+1(m \geq M)$. We

$$
\infty>\sum_{m \geq M} Y_{m}\left(d_{0}\right) T^{m}=\sum_{m \geq M} \frac{C_{m}\left(d_{0}\right)}{d_{0}^{m+g-M+1}} T^{m}=\frac{1}{d_{0}^{g-M+1}} \sum_{m \geq M} C_{m}\left(d_{0}\right)\left(\frac{T}{d_{0}}\right)^{m} .
$$

and if $|t / d|<\left|T / d_{0}\right|$ and $0<d \leq d_{0}$, then

$$
\begin{aligned}
u & \ll Y=\sum_{m \geq 1} Y_{m}(d) t^{m} \\
& =\sum_{1 \leq m \leq M-1} Y_{m}(d) t^{m}+\sum_{m \geq M} \frac{C_{m}(d)}{d^{m+g-M+1}} t^{m} \\
& =\sum_{1 \leq m \leq M-1} Y_{m}(d) t^{m}+\frac{1}{d^{g-M+1}} \sum_{n \geq M} C_{m}(d)\left(\frac{t}{d}\right)^{m} .
\end{aligned}
$$




$$
|u| \leqq \sum_{1 \leq m \leq M-1} Y_{m}(d)|t|^{m}+\frac{1}{d^{g-M+1}} \sum_{m \geq M} C_{m}\left(d_{0}\right)\left(\frac{T}{d_{0}}\right)^{m}<\infty .
$$

So for $x$ sufficiently close to the origin, there exists a positive constant $C$ such that $u(t, x)$ is holomorphic in $|t|<C d(x)$. (In fact $C=\left|T / d_{0}\right|$.)

Next, we consider the case $M<g+2$, where $t_{l M+k}=l(g+2)+k-1(l \geq 1$, $0 \leq k \leq M-1)$. We have

$$
\begin{aligned}
\infty>\sum_{m \geq M} Y_{m}\left(d_{0}\right) T^{m} & =\sum_{k=0}^{M-1} \sum_{l=1}^{\infty} \frac{C_{l M+k}\left(d_{0}\right)}{d_{0}^{l(g+2)+k-1}} T^{l M+k} \\
& =\sum_{k=0}^{M-1} \frac{T^{k}}{d_{0}^{k-1}} \sum_{l=1}^{\infty} C_{l M+k}\left(d_{0}\right)\left(\frac{T^{M}}{d_{0}^{g+2}}\right)^{l} .
\end{aligned}
$$

Hence if $\left|t^{M} / d^{g+2}\right|<\left|T^{M} / d_{0}^{g+2}\right|$ and $0<d \leq d_{0}$, then

$$
\begin{aligned}
u & \ll Y \\
& =\sum_{m \geq 1} Y_{m}(d) t^{m} \\
& =\sum_{1 \leq m \leq M-1} Y_{m}(d) t^{m}+\sum_{k=0}^{M-1} \frac{t^{k}}{d^{k-1}} \sum_{l=1}^{\infty} C_{l M+k}(d)\left(\frac{t^{M}}{d^{g+2}}\right)^{l}, \\
|u| & \leqq \sum_{1 \leq m \leq M-1} Y_{m}(d) \mid t^{m}+\sum_{k=0}^{M-1} \frac{t^{k}}{d^{k-1}} \sum_{l=1}^{\infty} C_{l M+k}\left(d_{0}\right)\left(\frac{T^{M}}{d_{0}^{g+2}}\right)^{l}<\infty
\end{aligned}
$$

So for $x$ sufficiently close to the origin, there exists a positive constant $C$ such that $\imath(t, x)$ is holomorphic in $\left|t^{M}\right|<C d(x)^{g+2}$.

The proof of the Main Theorem has now been completed.

\section{§3. Appendix}

We give the proof due to T. Oaku of the estimate (6) in the Introduction. The author is very much grateful for him.

Proposition 2. Let $f(x)$ be a holomorphic function defined in a neighborhood $\Omega$ of the origin of $\mathbb{C}_{x}^{n}, x=\left(x_{1}, \ldots, x_{n}\right)$. Assume that $f(x)$ has a zero exactly of order $g \in \mathbb{N}^{*}$ at the origin. Denote by $V$ the set $\{x \in \Omega ; f(x)=0\}$, and by $d(x)$ the distance from $x \in \Omega$ to $V$. Then there exist a neighborhood $\Omega^{\prime} \subset \Omega$ of the origin and a positive constant $C>0$ such that $|f(x)| \geq C d(x)^{g}, x \in \Omega^{\prime}$.

Proof.

Let the Maclaurin expansion of $f(x)$ be $f(x)=\sum_{|\alpha| \geq g} f_{\alpha} x^{\alpha}$. Set $f_{g}(x)=$ $\sum_{|\alpha|=g} f_{\alpha} x^{\alpha}$. which is a nonzero homogeneous polynomial. By applying a suitable linear change of coordinates if necessary, we may assume that $f_{(g, 0, \ldots, 0)} \neq$ 0 . The preparation theorem of Weierstrass implies that $f(x)$ can be written in the following form: 


$$
f(x)={ }_{c}(x)\left(x_{1}^{g}+a_{1}\left(x^{\prime}\right) x_{1}^{g-1}+\cdots+a_{g}\left(x^{\prime}\right)\right), \quad c(0) \neq 0, a_{1}\left(0^{\prime}\right)=\cdots=a_{g}\left(0^{\prime}\right)=0,
$$

where $c(x)$ is a holomorphic function defined near the origin 0 of $\mathbb{C}^{n}$ and each $a_{\mathfrak{l}}\left(x^{\prime}\right)(i=1,2, \ldots, g)$ is holomorphic near the origin $0^{\prime}$ of $\mathbf{C}_{x^{\prime}}^{n-1}, x^{\prime}=\left(x_{2}, x_{3}, \ldots\right.$, $x_{n}$ ). So there exist functions $\phi_{1}\left(x^{\prime}\right), \ldots, \phi_{g}\left(x^{\prime}\right)$ (which are not necessarily holomorphic) such that

$$
f(x)={ }_{c}(x) \prod_{j=1}^{g}\left(x_{1}-\phi,\left(x^{\prime}\right)\right), \quad \phi_{1}\left(0^{\prime}\right)=\cdots=\phi_{g}\left(0^{\prime}\right)=0
$$

If $x$ is sufficiently close to the origin, we have

$$
\begin{aligned}
|f(x)| & \geq \frac{1}{2}|c(0)| \prod_{j=1}^{g}\left|x_{1}-\phi_{j}\left(x^{\prime}\right)\right| \\
& \geq\left.\left.\frac{1}{2}\right|_{c}(0)\right|_{d}(x)^{g} .
\end{aligned}
$$

This kind of estimate does not hold in the real analytic case. We give a counterexample.

Set $f\left(x_{1}, x_{2}\right)=-x_{1}^{3}+x_{2}^{2},\left(x_{1}, x_{2}\right) \in \mathbb{R}^{2}$. The function $f$ has a zero of order 2 at $(0,0)$. Set $V^{\mathbf{R}}=\left\{\left(x_{1}, x_{2}\right) \in \mathbb{R}^{2}: f\left(x_{1}, x_{2}\right)=0\right\}$. If $d>0$ then the distance to $V^{\mathbf{R}}$ from the point $(-d, 0)$ is $d$. It is attained by $(0,0)$. Note that $f(-d, 0)$ $=d^{3}$.

This is not a paradox. There are some points in $V^{\mathbf{C}}=\left\{\left(z_{1}, z_{2}\right) \in \mathbb{C}^{2}\right.$; $\left.f\left(z_{1}, z_{2}\right)=0\right\}$ that are closer to $(-d, 0)$ than the origin is. Such points are found. for example, on the intersection of $V^{\mathbf{C}}$ and $\mathbb{R}_{x_{1}} \times i \mathbb{R}_{y_{2}} \subset \mathbb{C}_{\left(x_{1}+i y_{1}, x_{2}+i y_{2}\right)}^{2}=$ $\mathbb{C}_{\left(z_{1}, z_{2}\right)}^{2}$. In fact, the equation $f\left(x_{1}, i y_{2}\right)=-x_{1}^{3}-y_{2}^{2}=0$ defines a curve in $\mathbb{R}_{\left(x_{1}, y_{2}\right)}^{2}$ $\ni(-d, 0)$ which is very close to the point $(-d, 0)$ if $d>0$ is sufficiently small. below.

The reader is referred to [5] for estimates of real analytic functions from

\section{References}

[1] Gérard, R. and Tahara, H., Holomorphic and Singular Solutions of Nonlinear Singular First Order Partial Differential Equations, Publ. RIMS, Kyoto Univ., 26 (1990) . 979-1000.

[2] Singular Nonlinear Partial Differential Equations, Vieweg, 1996.

[3] Hille. E.. Ordinary differential equations in the complex domain, John Wiley and Sons, 1976.

[4] Kimura. T., Ordinary differentıal equations, Iwanami Shoten, 1977 (in Japanese).

[5] Lojasiewicz, S., Sur le problème de la divisıon, Studia Math., 18 (1959), 87-136. 
